\title{
Lafforgue Awarded 2019 Breakthrough Prize in Mathematics
}

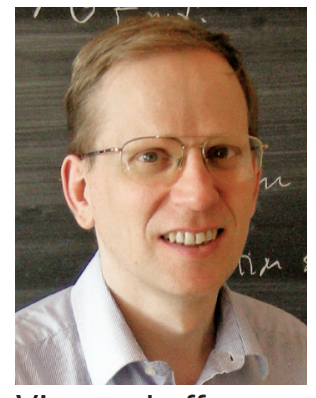

Vincent Lafforgue
VINCENT LAFFORGUE of the National Center for Scientific Research (CNRS) and Institut Fourier, Université Grenoble Alpes, has been awarded the 2019 Breakthrough Prize in Mathematics "for groundbreaking contributions to several areas of mathematics, in particular to the Langlands program in the function field case."

The prize committee provided the following statement: "The French lay claim to some of the greatest mathematical minds ever-from Descartes, Fermat and Pascal to Poincaré. More recently, Weil, Serre, and Grothendieck have given new foundations to algebraic geometry, from which arithmetic geometry was coined. Vincent Lafforgue is a leader of this latter school, now at the heart of new discoveries into cryptography and information security technologies. He makes his professional home in Grenoble at the CNRS, the largest fundamental science agency in Europe, where he has a tenured position and free rein to ponder the imponderable. Deeply concerned about the ecological crisis, Lafforgue is now focused on operator algebras in quantum mechanics and devising new materials for clean energy technologies."

"Vincent Lafforgue, in the so called 'function field case,' found a beautifully simple direct argument," said Richard Taylor, the chair of the selection committee. "After seeing it you ask yourself how the rest of us could have missed it for so long. You finally see why Langlands correspondence has to exist-it no longer seems an unmotivated miraculous consequence of complicated computations."

Lafforgue tells the Notices: "I am interested in maths for ecology and especially quantum mechanics for new materials for clean energy (because my first subject was operator algebras). I discovered that global public investment in

For permission to reprint this article, please contact: reprint -permission@ams.org.

DOI: https://dx.doi.org/10.1090/noti 1836 clean energy RD\&D is incredibly low: of the order of $0.02 \%$ of global GDP. Corporate investment is even lower, of the order of $0.01 \%$ of global GDP.

"Governments should really invest more in basic research and in RD\&D for clean energy (including nuclear energy, e.g., thorium molten salt reactors). Scientists can organize themselves by helping collaborations between mathematicians, physicists, chemists, biologists on subjects useful for ecology: multidisciplinary institutes could be dedicated to this purpose.

"More modestly, I am interested in creating a website where physicists, chemists, biologists working on subjects useful for ecology could explain the mathematical problems they have."

\section{Biographical Sketch}

Vincent Lafforgue was born in Antony, France, in 1974. He received his PhD from Ecole Normale Supérieure (ENS) in 1999 under the supervision of Jean-Benôit Bost. His thesis work concerned the Baum-Connes conjecture, and in his research he introduced original Banach algebra techniques to solve new cases of the conjecture. His honors include the 2000 EMS Prize and the 2015 CNRS Silver Medal for his work on global fields of positive characteristics, which contributed significantly to the Langlands program. He was an invited speaker at the 2002 International Congress of Mathematicians in Beijing. He likes to spend his time hiking in foothills of the Alps.

\section{About the Prize}

The Breakthrough Prize in Mathematics was created by Mark Zuckerberg and Yuri Milner in 2013. It recognizes major advances in the field, honors the world's best mathematicians and supports their future endeavors, and aims to communicate the excitement of mathematics to the general public. Breakthrough Prize Foundation sponsors are Sergey Brin, Priscilla Chan and Mark Zuckerberg, Ma Huateng, Yuri and Julia Milner, and Anne Wojcicki. 
The prizewinners were recognized at a ceremony that was broadcast live on National Geographic, YouTube, and Facebook Live. The prize is accompanied by a cash award of US $\$ 3$ million. Previous winners of the Breakthrough Prizes are:

- 2015: Simon Donaldson, Maxim Kontsevich, Jacob Lurie, Terence Tao, and Richard Taylor

- 2016: Ian Agol

- 2017: Jean Bourgain

- 2018: Christopher Hacon and James McKernan

\section{New Horizons in Mathematics}

The New Horizons in Mathematics Prizes are awarded to promising early career researchers who have already produced important work in mathematics. The honorees for 2019 are:

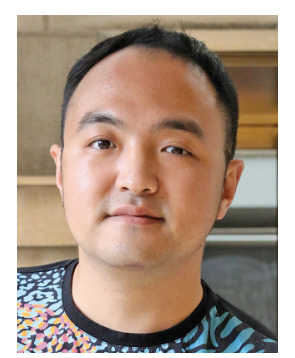

Chenyang $\mathrm{Xu}$

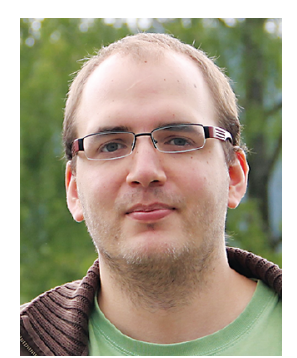

Karim Adiprasito

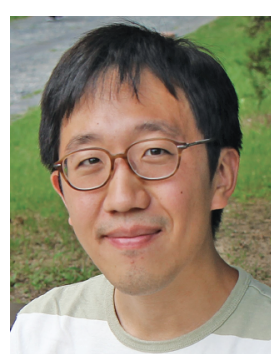

June Huh

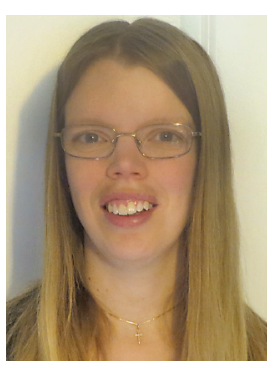

Kaisa Matomäki

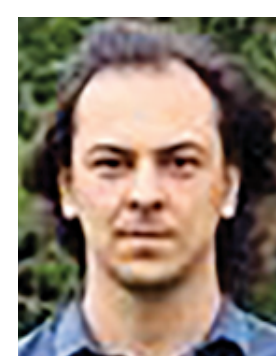

Maksym Radziwill
Chenyang Xu of the Massachusetts Institute of Technology and Beijing International Center for Mathematical Research "for major advances in the minimal model program and applications to the moduli of algebraic varieties."

$\mathrm{Xu}$ tells the Notices: "The best part of being a mathematician is that during the process of trying to solve a mathematical problem, I earn myself a peaceful mind. This becomes more and more important lately."

KARIM ADIPRASITO of the Hebrew University of Jerusalem and June HuH of the Institute for Advanced Study "for the development, with Eric Katz, of combinatorial Hodge theory leading to the resolution of the log-concavity conjecture of Rota."

KAISA MATOMÄKI of the University of Turku, Finland, and MAKSYM RADZIWILL of the California Institute of Technology "for fundamental breakthroughs in the understanding of local correlations of values of multiplicative functions."

\section{New Horizons in Mathematics}

The New Horizons in Mathematics Prizes are awarded to promising early career researchers who have already produced important work in mathematics. The honorees for 2019 are:

Chenyang Xu of the Massachusetts Institute of Technology and Beijing International Center for Mathematical Research "for major advances in the minimal model program and applications to the moduli of algebraic varieties."

$\mathrm{Xu}$ tells the Notices: "The best part of being a mathematician is that during the process of trying to solve a mathematical problem, I earn myself a peaceful mind. This becomes more and more important lately."

KARIM ADIPRASITO of the Hebrew University of Jerusalem and June HuH of the Institute for Advanced Study "for the development, with Eric Katz, of combinatorial Hodge theory leading to the resolution of the log-concavity conjecture of Rota."

KAISA МАTOMÄKI of the University of Turku, Finland, and MAKSYM RADZIWILL of the California Institute of Technology "for fundamental breakthroughs in the understanding of local correlations of values of multiplicative functions."

The prizes carry a cash award of US $\$ 100,000$. Collaborators share the award.

\section{-Elaine Kehoe with Breakthrough Prize Committee and CNRS announcements}

\section{Credits}

Photo of Vincent Lafforgue is by Gérard Laumon, CNRS. Photo of Chenyang Xu is by Allegra Boverman.

Photo of Karim Adiprasito is courtesy of Archives of the MFO.

Photo of June Huh is courtesy of Woo-Hyun Kim.

Photo of Kaisa Matomäki is by Pekka Matomäki.

Photo of Maksym Radziwill was provided by Petra Lein, () MFO. 